\title{
Suppressive Effect of Components in Lemon Juice on Blood Pressure in Spontaneously Hypertensive Rats
}

\author{
Yoshiaki MiyAKe, ${ }^{1}$ Keiko KuZuYA, ${ }^{1}$ Chisato Ueno, ${ }^{2}$ Naomi KatAyama, ${ }^{2}$ Takashi HayAKAwa, ${ }^{2}$ \\ Haruhito TSUGE ${ }^{2}$ and Toshihiko OSAWA ${ }^{3}$ \\ ${ }^{1}$ Central Research Laboratory of Pokka Corporation, Ltd., 45-2 Kumanosyo, Shikatsu-cho, Nishikasugai-gun, Aichi 481, Japan \\ ${ }^{2}$ Department of Food Science, Gifu University, Gifu 501-11, Japan \\ ${ }^{3}$ Department of Applied Biological Sciences, Nagoya University, Nagoya 464-01, Japan
}

Received May 28, 1997; Accepted September 10, 1997

\begin{abstract}
The effects of lemon juice and its crude flavonoids on blood pressure were examined using spontaneously hypertensive rats (SHR). The 5\% diluted lemon juice was orally administered in the diet to SHR, and they tended to have a lower systolic blood pressure than the control rats after 90 days. The systolic blood pressure of SHR fed a diet containing crude flavonoids from the juice for 16 weeks was significantly lower than that of the control group $(p<0.05)$. The systolic blood pressure with administration of fraction $B$, which was fractionated from the crude flavonoids, was significantly lower $(p<0.05)$ after 4 weeks. The crude flavonoids and fraction B contained abundant flavonoid glycosides of eriocitrin, hesperidin, and 6,8-di- $C$ - $\beta$-glucosyldiosmin as determined by high performance liquid chromatography (HPLC) analysis. The crude flavonoids and the flavonoid glycosides had an inhibitory effect on angiotensin I converting enzyme (ACE).
\end{abstract}

Keywords: lemon juice, blood pressure, spontaneously hypertensive rats (SHR), flavonoid

Lemon (Citrus limon BuRM. f.) is one of the most popular citrus fruits in the world, and the juice has been used in various food preparations, such as soft drinks, alcoholic drinks, seasonings, and candies. Lemon juice has been known as a typical healthy food for a long time, and it has been reported that it has a desmutagenic effect (Achiwa et al., 1991) and an antimutagenic effect (Jain et al, 1987). Lemon juice contains a number of nutrients such as citric acid, ascorbic acid, minerals, and flavonoids. Flavonoids that exhibit beneficial effects on capillary permeability and fragility were once known as vitamin P. The flavonoids in lemon fruit have been investigated with respect to many physiological functions (Middleton \& Kandaswami, 1994). We have found and reported antioxidative flavonoid compounds in lemon fruit (Miyake et al., 1997a, b). Flavonoid glycosides in lemon peel have also been reported to have a hypotensive effect (Kumamoto et al., 1984).

It had been reported that food materials such as tea catechins (Hara \& Tono-oka, 1990), cacao lignan (Kamiwaki et al., 1994), and wheat-brain hemicellulose (Kodama et al., 1996) had a suppressive effect on blood pressure in SHR. In this study, we paid much attention to the suppressive effect on blood pressure by orally administered feeding of lemon juice and its crude flavonoids. The effects of lemon juice and its crude flavonoids on blood pressure were examined in SHR. Those were also examined in vitro for the inhibitory effect on $\mathrm{ACE}$, in relation to the anti-hypotensive effect.

\footnotetext{
Materials and Methods

Materials The lemon juice used was a commercial material. Angiotensin-converting enzyme (ACE) from rabbit
}

lung and Hip-His-Leu were purchased from Wako Pure Chemical Industries, Ltd. (Osaka). Flavonoid glycosides and $(-)$-epigallocatechin gallate (EGCg) were obtained from Funakoshi, Ltd. (Tokyo). Eriocitrin and 6,8-di- $C$ - $\beta$-glucosyldiosmin were purified according to the method of Miyake et al. (1997a, b).

Preparation of crude flavonoid fractions from lemon juice Lemon juice $(3.0 l)$ was centrifuged at $4500 \times g$ for 15 min to remove the pulp. The supernatant was chromatographed on a Cosmosil 75C 18-OPN ODS column (Nacalai Tesque, Inc., Kyoto, $\phi 37 \times 500 \mathrm{~mm}$ ). The column was washed with $2 l$ of water and successively eluted with $2 l$ of $100 \%$ methanol. The elute portion was concentrated under reduced pressure and the crude flavonoids $(1.01 \mathrm{~g})$ were prepared. They were dissolved in $500 \mathrm{ml}$ water and centrifuged at $2000 \times \mathrm{g}$ for $20 \mathrm{~min}$ to remove precipitates. After being chromatographed on the same column as above, the column was washed with $2 l$ of water and successively eluted with 2 $l$ of $15 \%$ methanol and $2 l$ of $40 \%$ methanol. These eluted fractions were concentrated under reduced pressure. Fraction A (36.0 mg) and Fraction B (258 mg) were prepared. The samples for administration to rats, crude flavonoids, fraction $\mathrm{A}$, and fraction B, were dissolved in $1.0 \mathrm{l}$ of distilled water.

Experimental design SHR weighing 50 to $70 \mathrm{~g}$ (male 4-week-old SHR/NCrj, Charles River Japan, Ltd., Yokohama) were divided into four dietary groups of 5 animals each. The animals were fed a basal diet (MF powder, Oriental Yeast Co., Ltd., Tokyo). The SHR were housed in individual wire cages in a temperature-controlled room at $22^{\circ} \mathrm{C} \pm 1{ }^{\circ} \mathrm{C}$ with a 12-h cycle of light and dark. A nine-day training period was employed to acclimatize animals to the test 
environment. For experiment 1 , the rats were given the diets and a drinking solution supplemented with distilled water (control) and a solution of $5 \%$ diluted lemon juice ad libitum for 90 days. The intake of daily food and these solutions and the body weight were measured every 2 days. For experiment 2 , the rats were given the diets and a drinking solution supplemented with distilled water (control), a solution of crude flavonoids which was prepared with $1.01 \mathrm{~g}$ crude flavonoids in $1 l$ of distilled water, a solution of fraction $\mathrm{A}$ which was prepared with $36 \mathrm{mg}$ fraction $\mathrm{A}$ in $1 l$ of distilled water, and a solution of fraction B which was prepared with $258 \mathrm{mg}$ fraction $\mathrm{B}$ in $1 \mathrm{l}$ distilled water ad libitum for 16 weeks. The intake of daily food and these solutions and the body weight were measured every day.

Blood pressure measurement The blood pressure was measured by means of a monitor using a tail cuff (model BP-98A, Soften, Ltd., Tokyo). Values of the systolic, mean, and diastolic blood pressure for each conscious rat were determined by a tail-pulse pick-up method. The blood pressure values expressed are the means of 3 consecutive determinations. This measurement was performed during the period from noon to 6:00 p.m.

Identification of flavonoid glycosides The flavonoid compounds were identified by HPLC analysis (LC-10A, Shimadzu Co., Ltd., Kyoto) according to the method of Miyake et al. (1997b). HPLC was carried out using a Shim-pack CLC-ODS(M) $(4.6 \times 250 \mathrm{~mm})$ with a UV detector $(\lambda=280 \mathrm{~nm})$, at a $1.0 \mathrm{ml} / \mathrm{min}$ flow rate at $40^{\circ} \mathrm{C}$. The solvent system contained $70 \%$ of a $5 \%$ acetic acid solution and $30 \%$ methanol.

Assay of inhibitory effect of $A C E$ The assay of ACE activity was done according to the method of Horiuchi $e t$ al. (1982). The samples (crude flavonoids, fraction A, and fraction $\mathrm{B}$, and flavonoid glycosides) were dissolved in dimethyl sulfoxide and were added to reaction system. The inhibition concentration $\left(\mathrm{IC}_{50}\right)$ of the sample, which inhibits $50 \%$ of ACE activity, was evaluated. EGCg was used as the standard sample. A $50-\mu 1$ aliquot of diluted ACE was incubated in a total volume of $250 \mu \mathrm{l}$ of $130 \mathrm{mM}$ borate buffer (pH 8.3) containing $400 \mathrm{mM} \mathrm{NaCl}$ and $5 \mathrm{~mm}$ Hip-His-Leu for $30 \mathrm{~min}$ at $37^{\circ} \mathrm{C}$. The reaction was terminated by adding $0.75 \mathrm{ml}$ of $3 \%$ metaphosphoric acid and the mixture was centrifuged at $20,000 \times g$ for $5 \mathrm{~min}$. The hippuric acid released from Hip-His-Leu in the resultant supernatant was determined using HPLC, which was carried out using the above same column with a UV detector $(\lambda=228 \mathrm{~nm})$, at a $1.0 \mathrm{ml} /$ min flow rate at $40^{\circ} \mathrm{C}$.

Statistics Statistical analyses were performed using Duncan's multiple range test. $p$-values of less than 0.05 are considered to be of statistical significance.

\section{Results and Discussion}

Blood pressure At first, we attempted to administer $100 \%$ lemon juice orally to the SHR to see the suppressive effect of lemon juice on the blood pressure. However, the SHR drank little $100 \%$ lemon juice. The 5\% diluted lemon juice was drunk as well as the distilled water of the control group. Therefore, we examined the influence on the blood pressure of SHR by giving $5 \%$ lemon juice orally for 90 -days.
There was no significant difference between the total intake amount of the control group that was administered distilled water $(2540 \pm 259 \mathrm{~g})$ and that of the $5 \%$ lemon juice group that was administered $5 \%$ lemon juice $(2420 \pm 157 \mathrm{~g})$. No significant difference was observed between the control and the lemon juice groups in body weight gain, liver weight, and food intake. After the 90-day feeding period, the systolic blood pressure of rats fed $5 \%$ lemon juice was lower than that of the control but there was no significant difference $(p<0.05)$ between the two groups (Fig. 1). The rats orally administered with $5 \%$ lemon juice tended to show a suppressed increase in blood pressure. The flavonoid glycosides in lemon peel had been reported to have a hypotensive effect on blood pressure in SHR, which were intravenously injected with flavonoid glycosides (Kumamoto et al., 1984). We estimated whether the flavonoid compounds in lemon juice might have a suppressive effect on the blood pressure of SHR after oral feeding. We attempted to examine the suppressive effect on blood pressure in SHR following oral feeding with lemon flavonoids.

As shown in Materials and Methods, the crude flavonoids $(1.01 \mathrm{~g})$ were prepared from lemon juice $(3.00 \mathrm{l})$. Fraction A $(36.0 \mathrm{mg})$ and Fraction B $(258 \mathrm{mg})$ were prepared from the crude flavonoids using an ODS column. Each sample solution of the crude flavonoids, fraction $\mathrm{A}$, and fraction $\mathrm{B}$ was orally administered to the SHR for 16 weeks. No significant differences were observed between the four groups (control, crude flavonoids, fraction A, fraction B) in body weight gain, liver weight, and food intake. There was no significant

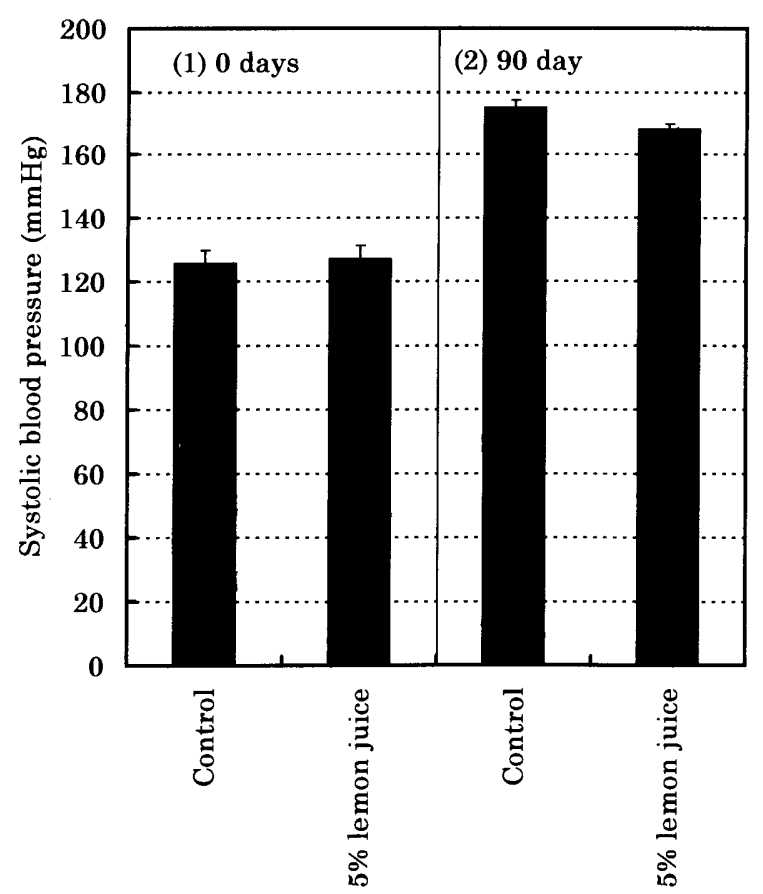

Fig. 1. The effect of lemon juice on blood pressure in SHR. (1) The systolic blood pressure of the control group and the diluted 5\% lemon juice group before administration. (2) The systolic blood pressure of the control group and the diluted 5\% lemon juice group after administration for 90 days. Each point represents the mean $\pm \mathrm{SE}$ for 5 rats. There is no significant difference between the water and $5 \%$ lemon juice groups. 
difference of the total intake amount among the control group $(3150 \pm 141 \mathrm{~g})$ that was administered distilled water, the crude flavonoids group $(3130 \pm 107 \mathrm{~g})$ that was administered a solution of crude flavonoids $(1.01 \mathrm{~g} / l)$, the fraction A group $(3370 \pm 138 \mathrm{~g})$ that was administered a solution of fraction $\mathrm{A}$ $(36.0 \mathrm{mg} / \mathrm{l})$, and the fraction B group $(3350 \pm 156 \mathrm{~g})$ that was administered a solution of fraction B $(258 \mathrm{mg} / l)$. The mean systolic blood pressure of SHR fed a diet containing crude flavonoids or fraction $\mathrm{B}$ for 16 weeks increased with time but remained significantly lower than that in the control group (Fig. 2). After the 16-week feeding period, the systolic blood pressure of the crude flavonoids group was significantly lower $(p<0.05)$ than that of the control, but the pressure of the group administered the fraction A was similar to that of the control. However, the systolic blood pressure of the fraction B group was significantly lower $(p<0.05)$ than that of the control after the 4 -week feeding period. The rats administered fraction B exhibited a stronger suppressive effect on blood pressure in SHR than the rats administered crude flavonoids. It is suggested that fraction B contains the component responsible for the suppressive effect on blood pressure.

Identification of flavonoid glycosides in crude flavonoid fraction The administered solutions of crude flavonoids, fraction $\mathrm{A}$, and fraction $\mathrm{B}$ were examined for the content of flavonoid glycosides. The nine flavonoid glycosides, eriocitrin, neoeriocitrin, narirutin, naringin, hesperidin, neohesperidin, diosmin, 6,8 -di- $C$ - $\beta$-glucosyldiosmin, and $6-C-\beta$ glucosyldiosmin in the fractions were identified by HPLC according to the method of Miyake et al. (1997b) (Table 1). Figure 3 shows the HPLC profiles of the administered solution of crude flavonoid, fraction $\mathrm{A}$, and fraction $\mathrm{B}$. The administered solution of crude flavonoids and fraction B which had a suppressive effect on blood pressure contained abundant eriocitrin, hesperidin and 6,8 -di- $C$ - $\beta$-glucosyldiosmin. The flavonoid compounds in HPLC profile were the main peak. The content of these compounds in the non-effective fraction A was very low. Some compounds other than flavonoid compounds seemed to exist in the solution of crude flavonoid and fraction $\mathrm{B}$, but these were suggested to be minor. Several papers have already been published on the hypotensive effects of flavonoid glycosides from the peels of Citrus group fruits. $6,8-\mathrm{Di}-C$ - $\beta$-glucosyldiosmin had been shown to have a high activity of a hypotensive effect on blood pressure in SHR (Kumamoto et al. 1984; 1985). Flavonoids such as rutin and hesperidin that exhibit beneficial effects on capillary permeability and fragility were once known as vitamin P. Hesperidin, the most important flavanone of Citrus sp., obtained from the solid residue of orange peel, showed an antihypertensive effect on SHR (Galati et al., 1996). 6,8-Di- $C$ - $\beta$-glucosyldiosmin and hesperidin in crude flavonoids and fraction $\mathrm{B}$ were indicated to have a suppressive effect on the blood pressure of SHR.

The ACE inhibitory effect of crude flavonoid fractions and flavonoid glycosides The renin-angiotensin system is of importance in regulating the blood pressure and the pathogenesis of hypertension. This system is also found in diverse tissues such as blood vessel walls, brain, and heart as well as the circulatory system, although their physiological roles are different (Matsufuji et al., 1995). A favorable antihypertensive drug is one that is sufficiently effective without any combined therapy and that has minimal side effects, while preventing or improving disorders in the organs including the kidney. From this point of view, ACE inhibitors are generally used for clinical cases of renal hypertension. Crude catechins in green tea had been examined for the inhibition of ACE activity to estimate their suppressive effect on blood pressure in SHR, and the EGCg of the major green tea polyphenolic component has been shown to exhibit noticeable inhibition of ACE activity (Hara et al, 1987). Thus, we examined the inhibition of ACE activity by crude flavonoid

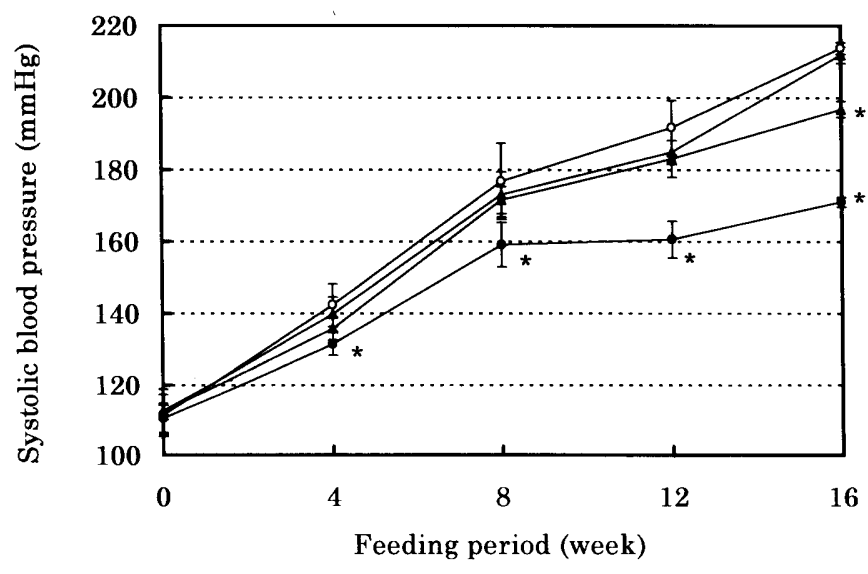

Fig. 2. The effect of crude flavonoid fractions on blood pressure of SHR. The line with opened circles $(\bigcirc)$, closed triangles $(\Delta)$, opened triangles $(\Delta)$ and closed circles $(\bullet)$ represents respectively the control group that was administered water, the crude flavonoids group that was administered a solution of crude flavonoids $(1.01 \mathrm{~g} / l)$, the fraction A group that was administered a solution of fraction A $(36.0 \mathrm{mg} / l)$, and the fraction B group that was administered a solution of fraction $B(258 \mathrm{mg} / l)$. Each point represents the mean \pm SE for 5 rats. There is a significant difference from the control group $\left({ }^{*} p<0.05\right)$.

Table 1. The identification of flavonoid glycosides in administered solution of crude flavonoids, fraction A, and fraction B.

\begin{tabular}{|c|c|c|c|c|c|c|c|c|c|}
\hline & \multicolumn{9}{|c|}{ Concentration (ppm) } \\
\hline & ERI & HES & DGD & DIO & NAR & NAN & GD & NeoERI & NeoHES \\
\hline Crude flavonoids & 122 & 68.1 & 26.1 & 10.2 & 6.0 & 3.6 & 2.4 & trace & trace \\
\hline Fraction A & 1.0 & $\operatorname{trace}^{a)}$ & 1.0 & trace & trace & $\mathrm{ND}^{b)}$ & trace & $\mathrm{ND}$ & ND \\
\hline Fraction B & 161 & 42.6 & 32.4 & 3.0 & 7.5 & 5.1 & 3.3 & trace & trace \\
\hline
\end{tabular}

a) less than $1.0 \mathrm{ppm}$.

${ }^{b)}$ not detected.

ERI: eriocitrin, NeoERI: neoeriocitrin, NAR: narirutin, NAN: naringin, HES: hesperidin, NeoHES: neohesperidin, DIO: diosmin, DGD: $6,8-\mathrm{di}-C$ - $\beta$-glucosyldiosmin, GD : 6- $C$ - $\beta$-glucosyldiosmin. 
(1)

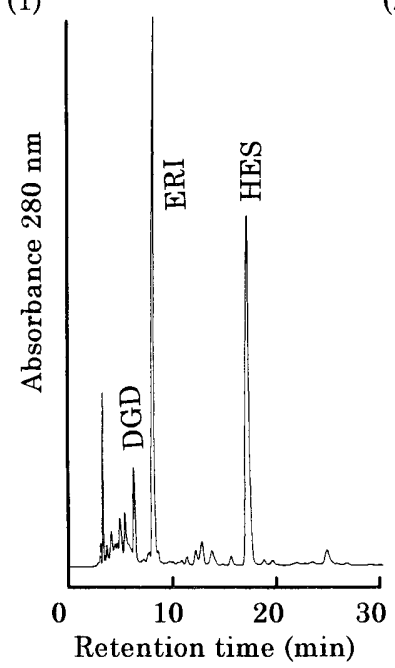

(2)

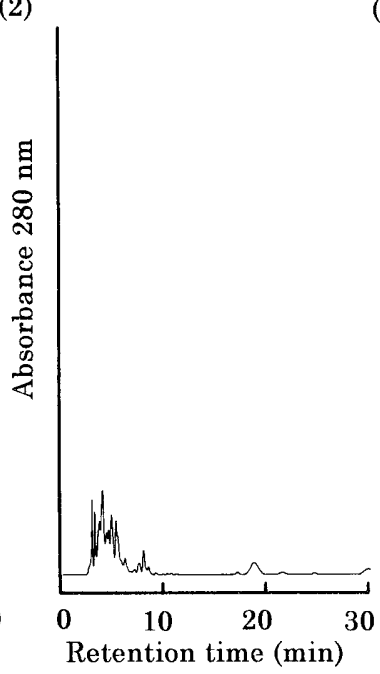

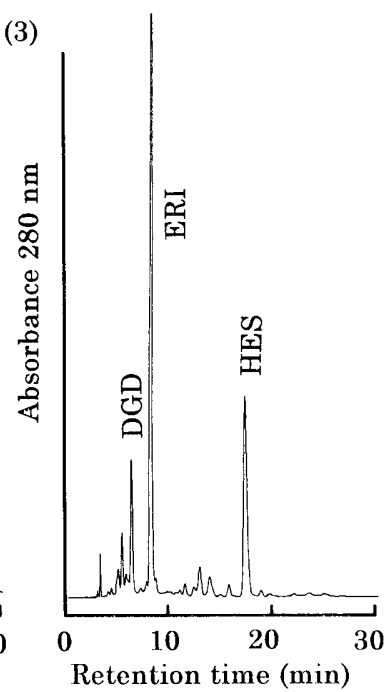

Fig. 3. HPLC profile of administered solution of crude flavonoids, fraction A, and fraction B. (1) The solution of crude flavonoid (1.01 g/ $l$ ) (2) The solution of fraction A $(36.0 \mathrm{mg} / l)(3)$ The solution of fraction B $(258 \mathrm{mg} / l)$. DGD: 6,8 -di- $C$ - $\beta$-glucosyldiosmin, ERI: eriocitrin, HES: hesperidin

Table 2. The ACE inhibitory effect of crude fractions and flavonoid glycosides.

\begin{tabular}{lc}
\hline & $\mathrm{IC}_{50}$ \\
\hline Crude flavonoids $(\mu \mathrm{g} / \mathrm{ml})$ & 20 \\
Fraction A & 100 \\
Fraction B & 2.5 \\
Flavonoid glycosides $(\mu \mathrm{M})$ & \\
Eriocitrin & 170 \\
Hesperidin & 100 \\
6,8-di- $C$ - $\beta$-glucosyldiosmin & 300 \\
$(-)$-epigallocatechin gallate & 150 \\
\hline EGCg $\cdot(-)$-epigallocatechin gallate, DGD $: 6,8$-di- $C$ - $\beta$-glucosyldiosmin.
\end{tabular}

fractions, which were prepared from lemon juice, and flavonoid glycosides in lemon juice (Table 2). Crude flavonoids, fraction A, and fraction B exhibited an inhibitory effect on ACE. Fraction B had an especially stronger activity than the other samples. The flavonoid glycosides of hesperidin, eriocitrin, and 6,8-di- $C$ - $\beta$-glucosyldiosmin had ACE inhibitory activity. Hesperidin exhibited stronger inhibitory activity on ACE than that of EGCg. The crude flavonoids contained abundantly the flavonoid glycosides of hesperidin, eriocitrin, and 6,8-di- $C$ - $\beta$-glucosyldiosmin as well as fraction B (Table 1 ), but the ACE inhibitory effect of crude flavonoid was weaker than that of fraction $\mathrm{B}$. The other components except for flavonoid glycosides in the fraction of crude flavonoids might suppress the ACE inhibitory effect. We suspected that these components were the water-insoluble compounds, which were removed in the process of purification of fraction $\mathrm{B}$, in the fraction of crude flavonoids. The flavonoid glycosides having an ACE inhibitory effect might function by a suppressive effect on blood pressure in SHR. The mechanism of the suppressive effect on blood pressure in vivo seems to require further examination.

\section{References}

Achiwa, Y., Koda, T. and Namiki, K. (1991). Inhibition of N-nitrosomorpholine formation by lemon juice. Nippon Shokuhin Kogyo
Gakkaishi, 38, 826-830 (in Japanese).

Galati, E.M., Trovato, A., Kirjavainen, S., Forestieri, A.M., Rossitto, A. and Monforte, M.T. (1996). Biological effects of hesperidin, a citrus flavonoid. (note III): Antihypertensive and diuretic activity in rat. IL Farmaco, 51, 219-221.

Hara, Y., Matsuzaki, T. and Suzuki, T. (1987). Angiotensin I converting enzyme inhibiting of tea components. Nippon Nogeikagaku Kaishi, 61, 803-808 (in Japanese).

Hara, Y. and Tono-oka, F. (1990). Hypotensive effect tea catechins on blood pressure of rats. Nippon Eiyo Syokuryo Gakkaishi, 43, 345348 (in Japanese).

Horiuchi, M., Fujimura, K., Terashima, T, and Iso, T. (1982). Method for determination of angiotensin-converting enzyme activity in blood and tissue by high-performance liquid chromatography. $J$. Chromatogr., 233, 123-130.

Jain, A.K., Shimoi, K., Nakamura, Y., Tomita, I. and Koda, T. (1987). Preliminary study on the desmutagenic and antimutagenic effect of some natural products. Curr. Sci., 56, 1266-1269.

Kamiwaki, T., Tsuji, K. and Nakagawa, Y. (1994). Effects of cacao lignin on blood pressure and lipid metabolism in spontaneously hypertensive rats. Nippon Nogeikagaku Kaishi, 68, 957-965 (in Japanese).

Kodama, T., Shiiba, K. and Tsuji, K. (1996). Suppressive effect of wheat-bran hemicellulose on blood pressure in spontaneously hypertensive rats. Nippon Eiyo Syokuryo Gakkaishi, 49, 101-105 (in Japanese).

Kumamoto, H., Matsubara, Y., Iizuka, Y., Murakami, T., Okamoto, K., Miyake, H. and Yokoi, K. (1984). Structure and hypotensive effect of flavonoids glycosides in lemon peeling. Nippon Nogeikagaku Kaishi, 58, 137-143 (in Japanese).

Kumamoto, H., Matsubara, Y., Iizuka, K., Okamoto, K. and Yokoi, K. (1985). Structure and hypotensive effect of flavonoid glycosides in lemon peelings (II). Nippon Nogeikagaku Kaishi, 59, 667-682 (in Japanese).

Matsufuji, H., Matsui, T., Ohshige, S., Kawasaki, T., Osajima, K. and Osajima, Y. (1995). Antihypertensive effects of angiotensin fragments in SHR. Biosci. Biotech. Biochem., 59, 1398-1401.

Middleton, E. and Kandaswami, C. (1994). Potential health-promoting properties of Citrus flavonoids. Food Tecnol, 48, 115-119.

Miyake, Y., Yamamoto, K. and Osawa, T. (1997a). Isolation of eriocitrin (eriodictyol 7-rutinoside) from lemon fruit (Citrus limon Burm. f.) and its antioxidative activity. Food Sci. Technol. Int. Tokyo, 3, 84-89.

Miyake, Y., Yamamoto, K., Morimitsu, Y. and Osawa, T. (1997b). Characteristics of antioxidative flavonoid glycosides in lemon fruit (Citrus limon). Food Sci. Technol. Int. Tokyo, 4, 48-53. 